$2 \cdot 1$ (SD 1·1), $P<0 \cdot 0001$; Matsuda index (4.7 (sD 2.7) $v \cdot 6 \cdot 7$ (SD 3.9), $P<0 \cdot 001$ ) and had higher glucose levels (fasting glucose $85 \cdot 4$ (sD 6.6) mg/dl $v .82 \cdot 4(\mathrm{sD} 5 \cdot 8$ ) $\mathrm{mg} / \mathrm{dl}, P<0 \cdot 01$; $2 \mathrm{~h}$ glucose $91 \cdot 8$ (sD $15 \cdot 8) \mathrm{mg} / \mathrm{dl} v .85 \cdot 2$ (SD 14.6) $\mathrm{mg} / \mathrm{dl}$, $P<0.05$ and higher) than those of Group 2. Lifestyle intervention induced a comparable significant decrease in weight, waist circumference, fat mass, heart rate and ALT (and increase in fat-free mass) in the two groups. Unlike Group 1 who had a significant decrease in glucose and triglyceride levels, Group 2 had a significant increase in glucose and white blood cells values and triglycerides (NS) ( $\mathrm{s} \%$ statistically significant between groups).

Conclusions: (i) Insulin resistance is often not associated with metabolic alterations in young obese people; (ii) lifestyle intervention, at least in a short-term period, induces metabolic improvements when obese children are insulin resistant whereas it may paradoxically worsen insulin sensitivity, glucose tolerance and inflammation when they are insulin sensitive; (iii) this finding is similar to that described in adult obese and its path physiology remains to be clarified.

\title{
Evaluation of new obesity-related individual metabolic and vascular risk profiles: the STYJOBS/EDECTA resource
}

\author{
Verena Biedermann ${ }^{1}$, Gunter Almer ${ }^{1}$, Sieglinde Zelzer ${ }^{1}$, Carmen Bauer-Denk $^{1}$, \\ Andreas Meinitzer ${ }^{1}$, Florian Freytag ${ }^{1}$, Renate Horejsi ${ }^{1}$, Reinhard Möller ${ }^{1}$, \\ Wolfgang Schnedl ', Kerstin Hingerl' ${ }^{1}$, Karl Koschutnig ${ }^{1}$, Gernot Reishofer ${ }^{1}$, \\ Renate Kruschitz ${ }^{1}$, Marcel Scheideler ${ }^{1}$, Martie Truschnig-Wilders ${ }^{1}$, Sandra Wallner- \\ Liebmann ${ }^{1}$, Robert Gasser ${ }^{1}$, Gerwin Bernhardt ${ }^{1}$, Elisabeth Kraigher-Krainer ${ }^{1}$, \\ Harald Mangge ${ }^{1}$, Daniel Weghuber ${ }^{2}$, Sabine Ebner ${ }^{2}$, Barbara Kofler ${ }^{2}$, Waltraud Eder ${ }^{2}$, \\ Wolfgang Sperl ${ }^{2}$, Elisabeth Ardelt-Gattinger ${ }^{3}$, Susanne Ring-Dimitriou ${ }^{3}$, \\ Monika Siegrist ${ }^{4}$, Christina Holzapfel ${ }^{4}$, Martin Halle ${ }^{4}$, Helmut Langhof $^{5}$ and \\ Vasan S Ramachandran ${ }^{6}$ \\ ${ }^{1}$ Medical University of Graz, Austria: ${ }^{2}$ Paracelsus Private University, Salzburg, Austria, Salzburg University, \\ Salzburg, Austria: ${ }^{3}$ Technical University Munich, Munich, Germany: ${ }^{4}$ Obesity Rehabilitation Clinic Schönsicht, \\ Berchtesgaden, Germany: ${ }^{5}$ Boston University School of Medicine, The Framingham Heart Study, Boston, MA, USA: \\ ${ }^{6}$ Obesity Academy Austria (OAA), Austria
}

Obesity is dramatically increasing in the western world. Atherosclerosis and type 2 diabetes, major consequences of obesity, start early in life and result in potentially fatal CVD. The STYrian Juvenile OBesity Study (STYJOBS) is a prospective study to improve the understanding of the major pathologic sequels of obesity by investigation of the 'non-biased' early phase in juveniles. EDECTA (Early DeteCTion of Atherosclerosis) extends STYJOBS to young and middle aged adults up to the age of 55 years.

Aims: Identification of 'individual metabolic high-risk patterns' in obesity by linking lab parameters (e.g. adipokines, lipid subfractions, natriuretic peptides, oxidative 'stress' biomarkers, molecular genetics, epigenetics), individual adipose tissue topography (lipometry), early vascular changes (carotids sonography), lifestyle habits, sport performance diagnostics and clinical data. STYJOBS/EDECTA-Database.

To establish a large serum/plasma/DNA resource of obese probands, their parents, and normal weight age matched controls comprehending the whole preclinical phase from 0 to 55 years (juveniles and adults) for advanced risk research (e.g. molecular genetics, epigenetics, microRNAs) in obesity. STYJOBS/EDECTA-Bioresource. To improve the understanding of addiction-like behaviour in obesity (e.g. imaging by 3 Tesla NMR after visual stimulation, analysis of dopamine receptor expression, link insulin resistance - control of hedonic inputs, craving). To establish by these data new strategies for prevention of lifestyle-associated diseases. STYJOBS/ EDECTA-Prevention.

Currently, STYJOBS/EDECTA comprehends data and biomaterial of $\sim 900$ participants aged between 3 and 55 years. The cohort is extended up to an end size of $n 1600$ to achieve one of the largest data and biomaterial resources addressing the preclinical phase of major sequels of obesity such as type 2 diabetes, myocardial infarction, stroke and cancer. These data should give a new basis for an improved personalised prevention. A broad international interdisciplinary co-operational network of scientists is involved in the STYJOBS/EDECTA activities. 
Methods applied: Routine lab, biomarkers (ELISA, HPLC, mass spectrometry, etc.), broad spectrum of obesity-related biomarkers with focus on adipokines, genetics (DNA, RNA, microRNAs, mitochondrial haplo- types), oxidative/nitrosative stress markers, carotids sonography, intima-media thickness, complex anthropometry including adipose tissue topography (lipometry).

\title{
Childhood Obesity: a Belgian Update
} The Belgian situation: a public health challenge

\author{
Michèle Guillaume
}

Head of the Department Nutrition, Environment and Health at the School of Public Health of the Faculty of Medicine of the University of Liege

Introduction: Childhood overweight and obesity remain a growing epidemic worldwide. During the past decades, the prevalence of overweight or obesity in children has doubled or tripled in several countries (Wang and Lobstein 2006). In Europe, the overall prevalence followed the same trend but with marked differences between countries (Lobstein and Frelut 2003). Here we present an update of the Belgian situation with respect to the international figures, as well as a review of environmental risk factors. What are the prevalence figures in Belgium?

Available data is difficult for comparisons because the age of the population studied and the definition used are different. At a national level, data based on self-reported height and weight were obtained from the Health Behaviour in School-aged Children study (HBSC). Results from the 2005-2006 HBSC study (WHO Regional Office for Europe 2008) for the French Community indicate that the prevalence of overweight or obesity, according to the International Obesity Task Force (IOTF) definition, amount $10 \%$ in both sexes at the age of 11 years, but increase to $10 \%$ and $14 \%$ at 13 years and to $12 \%$ and $15 \%$ at 15 years in girls and boys, respectively. Results were slightly higher than in previous surveys, although differences were not significant. In the Flemish Community, the prevalence of overweight or obesity was smaller than in the French Community, being $8 \%$ in both sexes at 11 years, 10\% in both sexes at 13 years and $8 \%$ and $11 \%$ for girls and boys, respectively, at 15 years of age. At a regional level, data are available from several studies in which height and weight were measured. In the province of Liège, a retrospective study was conducted based on school health records of 1403 children in 2005-2006. The mean age was 7 years (range: $2 \cdot 3-14 \cdot 6$ years). Using the IOTF definition, the prevalence of overweight or obesity was $16 \cdot 6 \%$ in girls and $15.9 \%$ in boys, and the prevalence of obesity was $4 \cdot 0 \%$ and $4 \cdot 4 \%$, respectively. In comparison with a previous study conducted in 1985, the prevalence of overweight albeit doubled in two decades (Guillaume and Counet 2009). In the province of Hainaut, a study conducted in 2003 revealed that the prevalence of overweight or obesity (according to the IOTF definition) was $25.9 \%$ in girls and $23.3 \%$ in boys at the age of 11 years (Observatoire de la Santé du Hainaut 2006). Finally, in the province of Luxembourg, a study conducted in 2004 (using the IOTF definition) showed that the prevalence of overweight or obesity was $25.6 \%$ in girls and $24.7 \%$ in boys at the age of 11 years (Counet et al. 2006). International comparison: the 2005-2006 HBSC study, which involved forty-one countries and regions, showed that the prevalence of overweight and obesity in Belgium ranked in the middle of the other countries, for both girls and boys (from 6\%, as in Netherlands, Lithuania and Romania, to $29 \%$ or $31 \%$, in the USA and Malta). Abdominal obesity is a real threat to health because it is a recognized risk factor for type 2 diabetes and CVD. Although it can already be detected in the late childhood and adolescence, to date, only a few studies have been performed among youngsters. In Belgium, a study conducted in 2008-2009, including 784 children of a mean age 11.5 ( $\mathrm{SD} 0.5$ ) years in the province of Liège, indicated that the prevalence of abdominal obesity varied between $15.4 \%$ according to the US percentile curves and $48.4 \%$ with the percentile curves of Great Britain. The prevalence was significantly higher in girls than in boys. According to these data, abdominal obesity in adolescents represents a significant public health problem in Liège, although the development of Belgian-specific reference curves would be required.

Risk factors: Several risk factors, such as genetic variability, are not modifiable but others, such as lifestyle and environmental risk factors, can be changed with prevention programs. However, these risk factors must be considered in a global context, both family-related and socio-economic.

Conclusions: Childhood overweight and obesity are a public health challenge in Belgium as they are worldwide. They require the involvement of all the actors of the society: politicians, health professionals, educational institutions, families, etc. The management should be multidisciplinary, centred on the child or the adolescent and their families. Since 2009, a research program called 